\title{
Simulation of the Load-Unload Response Ratio and Critical Sensitivity in the Lattice Solid Model
}

\author{
Peter Mora, ${ }^{1}$ Yu Cang Wang, ${ }^{1,2}$ Can Yin, ${ }^{1}$ \\ David Place, ${ }^{1}$ and XIANG-CHU YIN ${ }^{2,3}$
}

\begin{abstract}
The Load-Unload Response Ratio (LURR) method is an intermediate-term earthquake prediction approach that has shown considerable promise. It involves calculating the ratio of a specified energy release measure during loading and unloading where loading and unloading periods are determined from the earth tide induced perturbations in the Coulomb Failure Stress on optimally oriented faults. In the lead-up to large earthquakes, high LURR values are frequently observed a few months or years prior to the event. These signals may have a similar origin to the observed accelerating seismic moment release (AMR) prior to many large earthquakes or may be due to critical sensitivity of the crust when a large earthquake is imminent. As a first step towards studying the underlying physical mechanism for the LURR observations, numerical studies are conducted using the particle based lattice solid model (LSM) to determine whether LURR observations can be reproduced. The model is initialized as a heterogeneous 2-D block made up of random-sized particles bonded by elastic-brittle links. The system is subjected to uniaxial compression from rigid driving plates on the upper and lower edges of the model. Experiments are conducted using both strain and stress control to load the plates. A sinusoidal stress perturbation is added to the gradual compressional loading to simulate loading and unloading cycles and LURR is calculated. The results reproduce signals similar to those observed in earthquake prediction practice with a high LURR value followed by a sudden drop prior to macroscopic failure of the sample. The results suggest that LURR provides a good predictor for catastrophic failure in elastic-brittle systems and motivate further research to study the underlying physical mechanisms and statistical properties of high LURR values. The results provide encouragement for earthquake prediction research and the use of advanced simulation models to probe the physics of earthquakes.
\end{abstract}

Key words: Numerical simulation, Load-Unload Response Ratio method, earthquake prediction, lattice solid model, critical sensitivity.

${ }^{1}$ QUAKES, Department of Earth Sciences, The University of Queensland, Brisbane, 4072, Qld, Australia. E-mails: mora@quakes.uq.edu.au; wangyc@quakes.uq.edu.au; canyon@quakes.uq.edu.au; place@quakes.uq.edu.au

${ }^{2}$ LNM, Institute of Mechanics, Chinese Academy of Sciences, Beijing, 100080, China.

E-mail: xcyin@public.bta.net.cn

${ }^{3}$ Center for Analysis and Prediction, China Seismological Bureau, Beijing, 100036, China.

E-mail: yinxc@btamail.net.cn 


\section{Introduction}

The Load-Unload Response Ratio (LURR) method is an intermediate-term earthquake prediction approach (YIN et al., 1995, 2000) that has shown considerable promise. The method typically involves calculating the ratio of Benioff strain release during periods of loading and unloading as determined by calculating earth tide induced perturbations in the Coulomb Failure Stress on optimally oriented faults. In retrospective studies, high LURR values have been observed months to years prior to most events and some intermediate-term earthquake predictions have been made (YIN et al., 2000).

The idea that motivated the LURR earthquake prediction approach is that when a system is stable, its response to loading is nearly the same as its response to unloading, whereas when the system is in an unstable state, the response to loading and unloading becomes quite different (YIN et al., 1995, 2000). LURR is defined according to this difference. Suppose $P$ and $R$ are respectively the load and response of a system. If $P$ undergoes a small change $\Delta P$ resulting in a small change to $R$ of $\Delta R$, then

$$
X=\lim _{\Delta P \rightarrow 0} \frac{\Delta R}{\Delta P},
$$

can be defined as the response rate, and LURR is defined as

$$
\text { LURR }=\frac{X^{+}}{X^{-}},
$$

where $X^{+}$and $X^{-}$are response rates during loading and unloading. When a system is in a stable or linear state, $X^{+} \approx X^{-}$so LURR $\approx 1$. When a system lies beyond the linear state, $X^{+}>X^{-}$and LURR $>1$. Hence, LURR can be used as a criterion to judge the degree of stability of a system.

In earthquake prediction practice using LURR, loading and unloading periods are decided by calculating the earth tide induced perturbations in the Coulomb Failure Stress on optimally oriented faults (or a specified fault plane orientation), and LURR is often defined as ratio of cumulative Benioff strain release during loading compared to unloading. Specifically,

$$
\operatorname{LURR}=B^{+} / B^{-} .
$$

where $B^{+}$and $B^{-}$, respectively denote the cumulative Benioff strain release during loading and unloading. To avoid violent fluctuations due to poor statistics, the LURR values are computed from the cumulative Benioff strain release during loading and unloading summed over many load-unload cycles within a specified sliding time window (i.e., there are generally few events during a single load-unload cycle which lead to large statistical fluctuations in LURR between successive load-unload cycles). The length of the time windows must be chosen such that $B^{+}$ 
and $B^{-}$include enough earthquake events to average out the statistical fluctuations although not so long as to remove any time-varying signals with a physical origin. Typically, time windows of weeks to months are used in practice. In retrospective studies, high values of LURR have been observed a few months or years prior to most of the events and intermediate-term earthquake predictions have been made using this method (YIN et al., 2000).

In recent years, accelerating seismic moment release (AMR) has been observed prior to many large earthquakes (BUFE and VARNES, 1993; BOwMAN et al., 1998). Both AMR and high LURR may have a similar origin (YIN et al., 2002) or LURR may be due to critical sensitivity before catastrophic events (WEI et al., 2000). A physically based numerical simulation that is capable of reproducing LURR signals would provide a means to study the underlying physical mechanism for LURR signals.

The Lattice Solid Model (LSM) was developed to provide a basis to study the physics of rocks and the nonlinear dynamics of earthquakes (MORA and PlaCE, 1994, 1998; Place and Mora, 1999, 2000, 2001; Place et al., 2001). The LSM consists of a lattice of interacting particles. Intact material is modelled as particles linked by elastic-brittle bonds which can break if the separation exceeds a given threshold $R_{b}$ relative to the equilibrium separation $R_{0}$, and frictional forces are applied to unbonded particles that come into contact. Using the LSM, fracture, shearing of rock, stick-slip behavior, dynamic rupture, and wave propagation are simulated with relative simplicity. Localization phenomena in fault gouge zones has been simulated (PLACE and MORA, 2000) and recent results have provided a comprehensive potential explanation for the Heat Flow Paradox (MORA and Place, 1998, 1999). Recent numerical experiments involving model systems subjected to compression have demonstrated that the LSM is capable of realistically simulating the fracturing behavior of rocks (PlaCe and Mora, 2001; PlaCe et al., 2002). The lattice solid calculations compute the energies within the system (kinetic energy, energy lost to the artificial viscosity, fracture energy, energy lost to friction, external work done and potential energy). Numerical studies have verified that the sum of these energies is numerically conserved to a high precision (PLACE and MORA, 1999). Since the LSM is capable of realistically modeling fracture and slip events, it provides a means to study the underlying mechanism of LURR. In the following, we conduct simulations using the LSM with the aim of determining whether LURR signals can be reproduced as a first step towards this goal.

\section{Numerical Experiments of LURR}

In the present study, the model is initialized as a heterogeneous 2-D block made up of random-sized particles with diameters ranging from 0.2 to 1 model units. The system is subjected to uni-axial compression from rigid driving plates on the upper and lower edges of the model. Snapshots from a typical simulation are shown in 

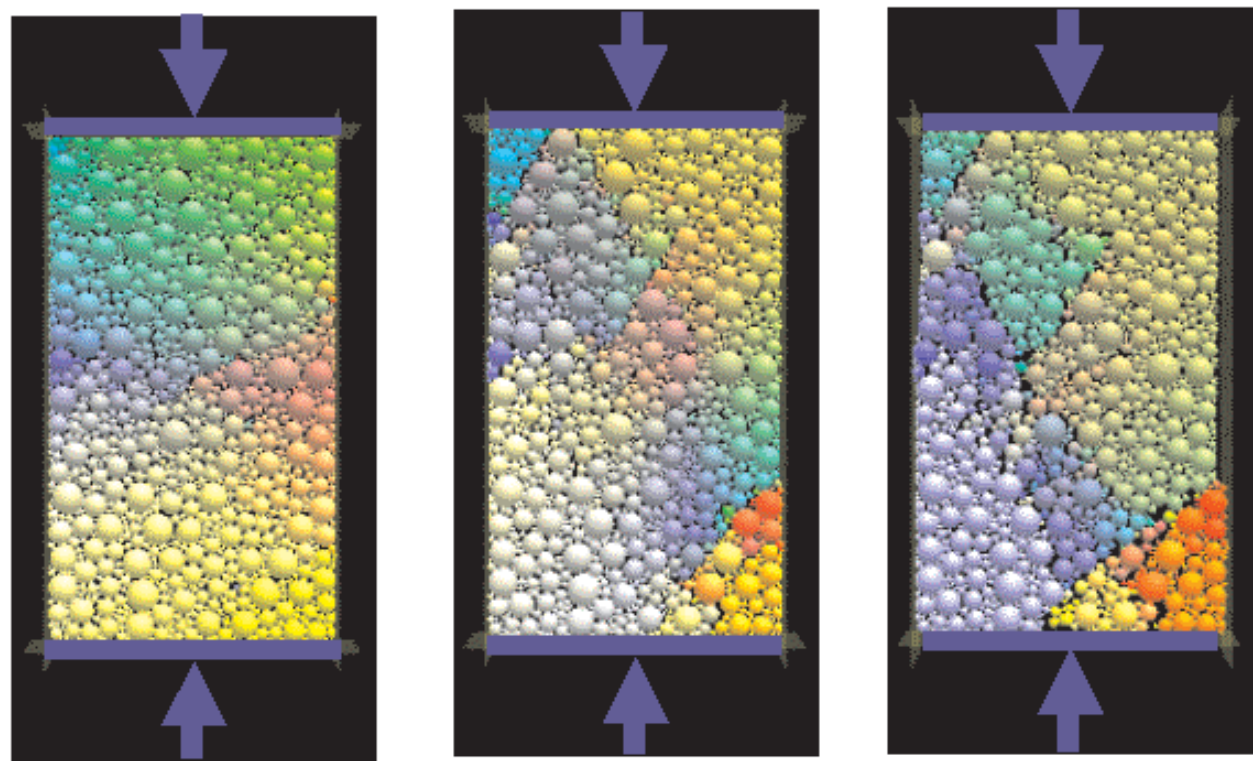

Figure 1

The setup for uniaxial compression numerical experiments illustrating formation of fractures in the random lattice solid model. Colors depict displacement (blue $=$ left, red $=$ right, green $=$ down, yellow $=$ up).

Figure 1. Two numerical experiments were conducted. In the first, loading is strain controlled and a constant driving rate is applied to the upper and lower edges of the model. In the second, stress control is used in which stress on the upper and lower edges is increased linearly and slowly until the sample fails. These two cases correspond to different effective rigidity outside the focal region and can yield different fracture behaviors. In both cases, a sinusoidal variation is added to the constant loading rate in order to simulate the stress perturbations induced by tidal forces. These variations resulted in stress of form

$$
\Delta \sigma_{z z}=a \sin (2 \pi t / T)
$$

so stress $\sigma_{z z}=\Delta \sigma_{z z}+k t$. The model initialization and parameters used in each experiment were identical. Tectonic loading is very slow. However, due to limited computer power, it is infeasible to use the observed tectonic driving rates in the numerical experiments. Therefore we use a higher loading rate $k \gg k_{\text {tectonic }}$. With the aim of ensuring the results remain meaningful with this higher loading rate, we selected parameters such that $\left|\Delta \sigma_{z z}\right| \ll \sigma_{z z}, \frac{d\left|\Delta \sigma_{z z}\right|}{d t} \gg k$ and $T_{e} \ll T \ll T_{L}$, where $T_{e}$ is the synthetic earthquake rupture duration and $T_{L}$ is the average time interval between large earthquakes. The values specified were loading rate $k=30 \mathrm{MPa} / 100,000$ time steps, period of the sinusoidal perturbations $T=4000$ time steps, amplitude of sinusoidal perturbation $a=0.96 \mathrm{MPa}=$ constant and breaking criterion 
$R_{b}=1.002 \mathrm{R}_{0}$. The elastic properties and model size were such that shear waves propagated vertically through the model in about 530 time steps, substantially shorter than period $T$. LURR values were calculated according to Equation (3) but using the cumulative energy release instead of cumulative Benioff strain release, i.e.,

$$
\operatorname{LURR}=E^{+} / E^{-},
$$

where $E^{+}$and $E^{-}$, respectively denote the cumulative seismic energy release during loading and unloading within a given time window. These were obtained by summing total kinetic energy released during all loading or unloading cycles within the specified time window, where we define loading to be when $\frac{d \sigma_{z z}}{d t} \geq 0$ and unloading when $\frac{d \sigma_{z z}}{d t}<0$. Specifically, the LURR value was calculated using

$$
\operatorname{LURR}(n)=\frac{E^{+}}{E^{-}}=\frac{\sum_{i=1}^{m} \Delta E^{+}(n-i+1)}{\sum_{i=1}^{m} \Delta E^{-}(n-i+1)},
$$

where $m$ is the number of cycles in the time window (i.e., the LURR time window length is $m T$ load-unload cycles), $n=m, m+1, m+2, \ldots$ is a time index (i.e., $t=n T$ ), and $\Delta E^{+}(j)$ and $\Delta E^{-}(j)$, respectively denote total kinetic energy released during the $j$-th load and unload cycle. The total kinetic energy release at any given instant $t$ is the sum of the kinetic energy within the system and the energy lost to the artificial viscosity prior to time $t$, i.e., $E_{\mathrm{TOTAL}}(t)=E_{k}(t)+E_{v}(t)$ where $E_{k}(t)$ denotes the kinetic energy at time $t$ and $E_{v}(t)$ denotes the energy lost to the artificial viscosity prior to time $t$. The total energy release for the $j$-th load or unload cycle was therefore calculated using

$$
\Delta E_{\mathrm{TOTAL}}^{+}(j)=E_{\mathrm{TOTAL}}\left(j T+T / 4+\Delta t_{k}\right)-E_{\mathrm{TOTAL}}\left(j T-T / 4-\Delta t_{k}\right),
$$

and

$$
\Delta E_{\text {TOTAL }}^{-}(j)=E_{\text {TOTAL }}\left(j T+3 T / 4-\Delta t_{k}\right)-E_{\text {TOTAL }}\left(j T+T / 4+\Delta t_{k}\right),
$$

where $\Delta t_{k}=T / 2 \pi \arcsin (k T / 2 a \pi)\left(\Delta t_{k}\right.$ takes into account the time difference between the loading and unloading periods due to the linearly increasing stress).

\section{Strain-controlled Compression Experiment}

Figure 2 shows stress, kinetic energy, LURR value and the total energy released versus time step for the strain-controlled experiment. In this experiment, LURR is calculated using a sliding time window that is ten load-unload cycles long with a sliding increment of one cycle. Hence, LURR is calculated from Equation (6) using $m=10$.

The sharp drops in stress and spikes of kinetic energy correspond to dynamic fracturing involving breaking of bonds and/or slip along fracture surfaces. These represent events in the simulation. Due to the artificial viscosity that is applied to 

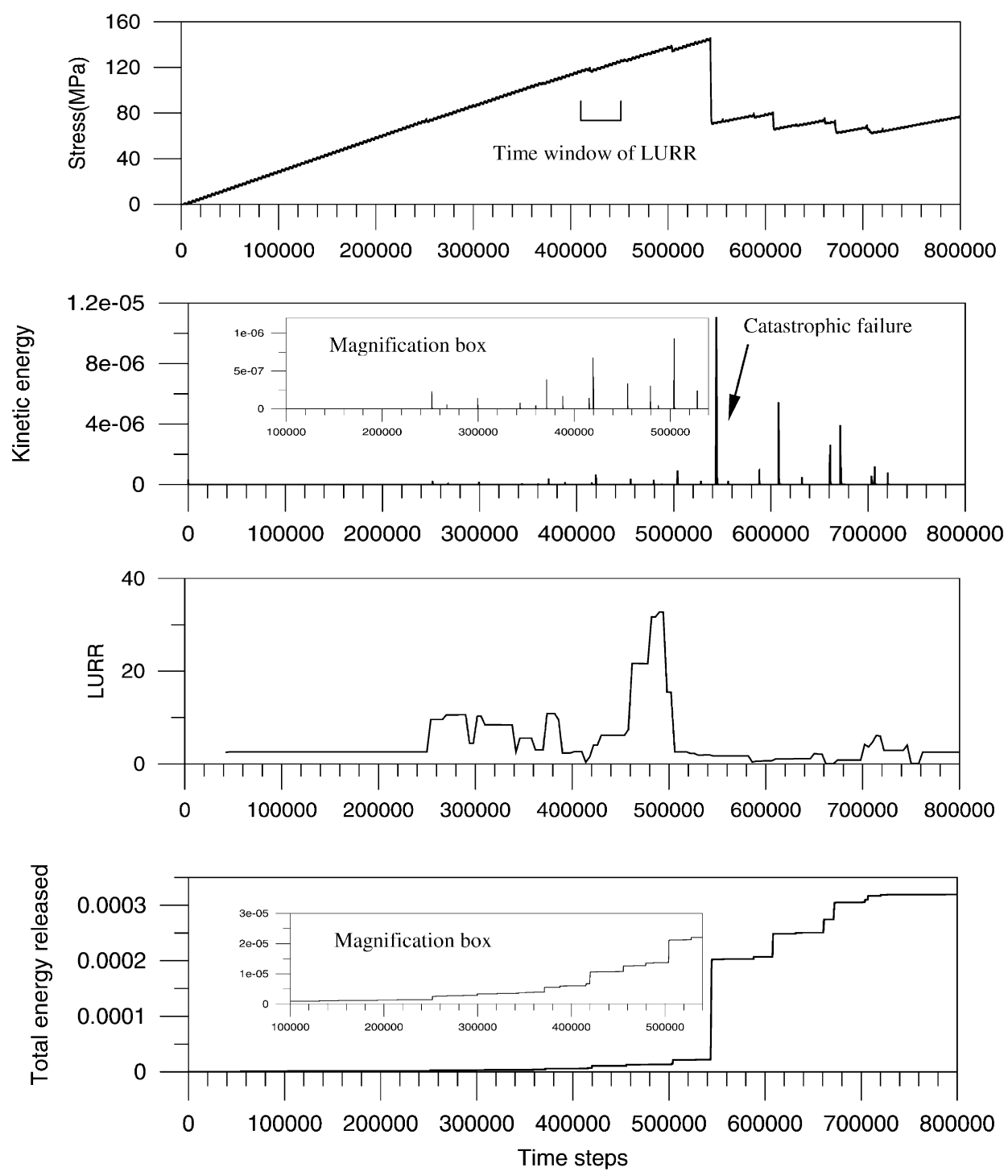

Figure 2

Results of the strain controlled experiment. From top to bottom, stress measured on the rigid driving plates, kinetic energy within the model, LURR value calculated from equations (6) through (8) and cumulative seismic energy release.

damp energy from the system, kinetic energy soon dies out after each event. It is this lost energy - termed viscous energy - summed with the instantaneous kinetic energy in the system that provides a measure of the total kinetic energy released by events $\left(E_{\text {TOTAL }}=E_{v}+E_{k}\right)$ and allows LURR to be computed using equations (6) through (8). 
The main rupture (catastrophic failure) of the sample occurred at 543,000 time steps and is seen as a sharp drop in stress, a large spike in kinetic energy, and a large step in total energy released. Just prior to failure, the stress reaches a peak of approximately $145 \mathrm{MPa}$ which corresponds to the strength of the sample. The LURR value remains constant until 250,000 time steps. During this period, the sample remained intact and so the viscous energy was small and equaled the energy lost to the viscosity in loading the system at the specified rate. Due to the loading, there is a small amount of kinetic energy which causes viscous energy to accumulate throughout the simulation even with no dynamic fracturing events. The total energy release due to this effect is not linearly increasing but has a periodic variation due to the sinusoidal stress perturbations. For this reason, the measured energy release during loading and unloading was not identical prior to the first fracture at 250,000 time steps resulting in an LURR value of 2.6 rather than unity. Between 250,000 and 340,000 time steps, small infrequent events occurred and the LURR value typically fluctuated between 4.5 and 10.5. Larger and more frequent events started to occur at around 370,000 time steps. After this time, the LURR value dropped and typically remained at around 2.5 to 8.5 until 460,000 time steps, with the exception of a short spike and trough. From 460,000 to 490,000 time steps, the LURR value rose significantly up to a peak value of 32 and then dropped again. This rise and subsequent drop in LURR occurred in several steps, indicating that the peak value was the result of high seismic energy release during several successive load-unload cycles. After 500,000 time steps, the LURR value dropped to a relatively low value of 2 despite continuing moderate-sized events and a large event at around 505,000 time steps. At 543,000 time steps, the catastrophic failure event occurred at which time the LURR value was 1.7. The LURR value continued to drop, reaching a low of 0.4 at 585,000 time steps. The LURR remained low for the remainder of the simulation despite several large subsequent ruptures.

Based on these results, it appears that the response of the system to loading and unloading becomes very different during a certain period just prior to the catastrophic failure and that high LURR values have successfully detected this critically sensitive state. Interestingly, the LURR value dropped just before the catastrophic failure event, suggesting that as the system becomes sufficiently damaged prior to the large event, it becomes insensitive to stress perturbations. A similar behavior of a sharp rise in LURR and a subsequent drop immediately before a large earthquake has also been observed in earthquake prediction practice (YIN et al., 2000).

\section{Stress-controlled Compression Experiment}

Figure 3 shows stress, kinetic energy, LURR value and total energy released versus time step for the stress-controlled experiment, where LURR is calculated from Equation (6) using $m=10$ as in the strain-controlled experiment. 

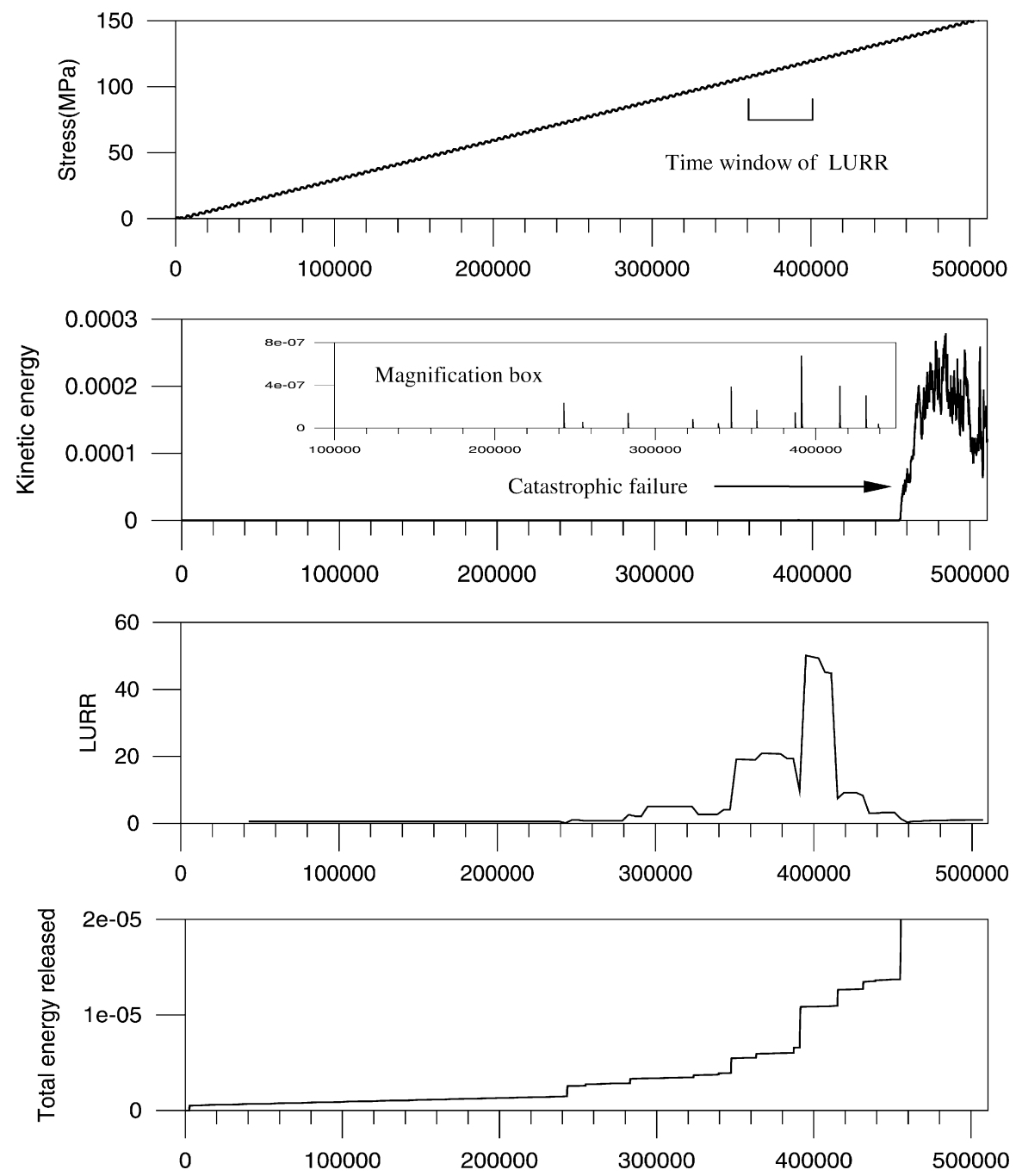

Figure 3

Results of the stress-controlled experiment. From top to bottom, stress measured on the rigid driving plates, kinetic energy within the model, LURR value calculated from equations (6) through (8) and cumulative seismic energy release.

The main rupture of the sample occurred at 455,000 time steps and is seen as a large rise in kinetic energy. Due to the use of stress control, the main rupture continues to grow and the sample fails catastrophically. Hence, kinetic energy does not drop once the main rupture occurs but remains at a high value. At the time of failure, the stress is around $137 \mathrm{MPa}$, somewhat lower than the peak stress in the strain-controlled experiment. The continued application of stress in this experiment probably explains the lower breaking strength of the sample than for the strain- 
controlled case. After a fracture event in the strain-controlled experiment, the internal configuration of the sample slightly changes as bonds break and surfaces slip, allowing the stress to drop and ruptures to arrest. As stress is built-up once again with continued strain loading, a new fracture will occur at the weakest point but due to the change in internal configuration, this may be in a different place than the previous fracture event. In contrast, the continued application of stress in the stresscontrolled experiment tends to enhance the possibility for a rupture to runaway catastrophically.

In the stress-controlled experiment (Fig. 3), the LURR value remains low at around 1 to 2 before 280,000 time steps (the stable period), then increases rapidly up to 50 prior to the catastrophic failure event. Even if event statistics are small, it is remarkable that the LURR value rose and then dropped prior to catastrophic failure, thereby exhibiting very similar behavior as in the strain-controlled case. This strongly suggests that events preferentially occur during loading compared to unloading when the system approaches an unstable state followed by a change in response when the unstable state is reached. These results imply that LURR is capable of detecting this unstable or critical state prior to catastrophic failure.

\section{Snapshots}

In order to visualize the development of fractures and damage, snapshots from the two simulations are shown in Figure 4. The initial fracture event in each case (respectively at around 250,000 and 243,000, time steps for strain and stress control) corresponds to fracturing that occurs near the upper left corner of the sample. Subsequent to this event, small events occur and the displacement field evolves as the system deforms. In the final image in each sequence, one clearly observes large offsets on several fractures in the failed system. The stress-controlled case is already highly failed at 455,000 time steps, and rapidly evolves to rubble at subsequent time steps.

\section{Discussion}

Many other simulations with different arrangements of random particles were also made and all results were similar to those presented. Although a full parameter study is beyond the scope of this paper, simulations were conducted using values $T, a$ and $k$ a factor of two larger and smaller. These tests yielded similar results to those presented here, suggesting insensitivity to the specific choice of loading period, stress perturbation amplitude and loading rate at least within the range studied.

Based on the simulation results, LURR is capable of detecting the critically sensitive or unstable state just prior to catastrophic failure of elastic-brittle systems. In the simulations, one observes a sharp rise in LURR in the lead-up to the main 


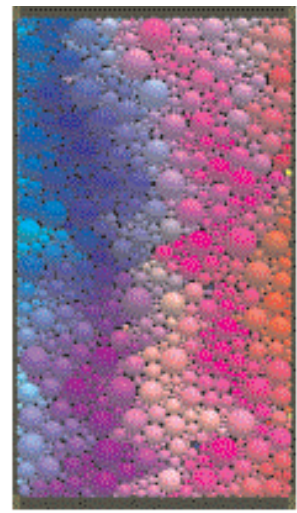

$t=250,000$

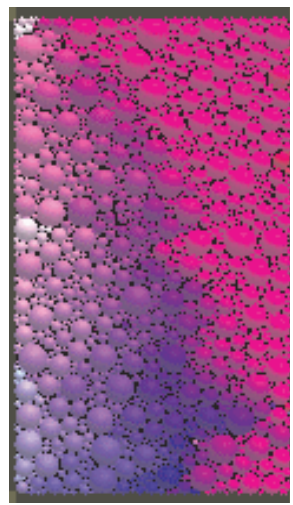

$\mathrm{t}=245,000$

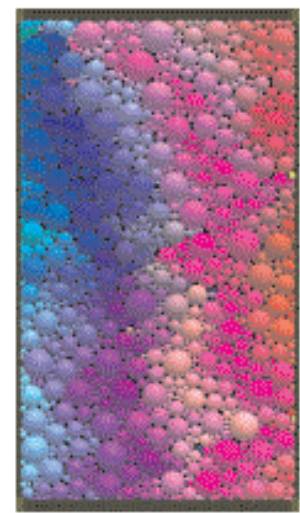

$t=255,000$

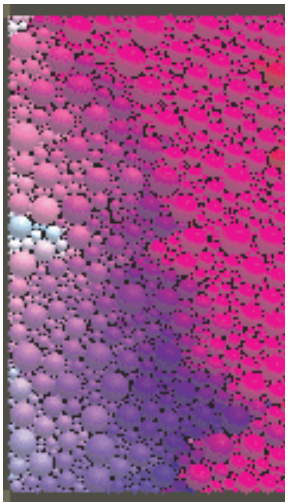

$\mathrm{t}=385,000$

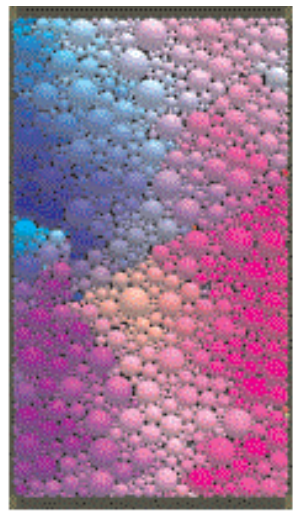

$t=540,000$

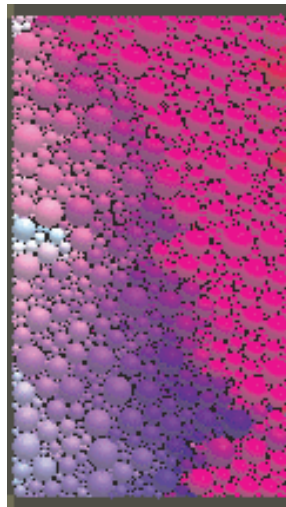

$\mathrm{t}=450,000$

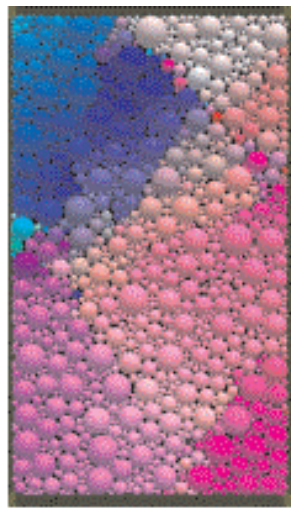

$t=545,000$

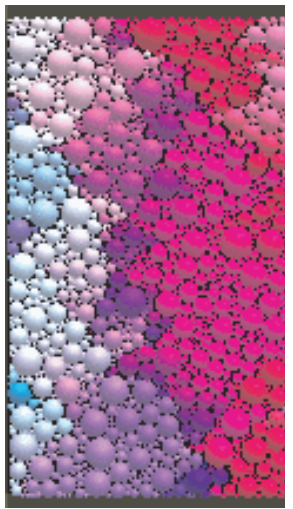

$\mathrm{t}=455,000$

Figure 4

Top: Snapshots from the strain-controlled experiment. Bottom: Snapshots from the stress-controlled experiment. Colors depict horizontal and vertical displacements. Images are scaled individually to enhance visualization of major features of the deformation field and fractures with large offsets.

event and then a drop shortly prior to catastrophic failure followed by low LURR values thereafter. The initial drop in LURR is caused by large events that occur during the unloading cycle or very close to the peak stress and spanning across the boundary into the unloading cycle. In both cases, the catastrophic failure occurs during the unloading cycle near the peak stress although we believe the typical case would be for it to occur at random during loading or unloading near the peak stress. The results suggest that the system becomes insensitive to stress perturbations after sufficient damage has occurred or after catastrophic failure of the system. This behavior of high LURR values in the lead-up to events and a drop immediately prior 
to an event is frequently observed in LURR earthquake prediction practice (YIN et al., 2000).

The results provide encouragement for earthquake forecasting research and the use of advanced simulation models to probe the physical mechanisms involved. Recent simulations exhibit AMR (Place and Mora, 2000; Mora and Place, 2002) and an evolution in stress correlations prior to large events consistent with the Critical Point Hypothesis for earthquakes (MorA and Place, 2002). Observational studies (YIN et al., 2002) showing that the critical scaling regions for AMR and LURR are identical suggest LURR has a common physical origin as AMR. If so, AMR may be detecting the lead-up to a critical point whereas LURR may detect critical sensitivity once the system is very close to, or has reached, the unstable regime or critical point.

\section{Conclusions}

The lattice solid model has been used to simulate a 2-D elastic-brittle system being subjected to uniaxial compression in which stress perturbations are superimposed on the otherwise constant strain or stress loading rates. In each case, fractures develop and seismic energy is radiated within the model as the system is compressed until the sample fails catastrophically. The Load-Unload Response Ratio (LURR) that has been used for intermediate-term earthquake predictions is calculated in each case from the seismic energy release in the model during loading and unloading. The results show that LURR values become high and then drop prior to the main event, and remain low thereafter. These results reproduce LURR signals similar to those that have often been observed in earthquake prediction practice and suggest that LURR is correctly identifying the critically sensitive or unstable regime prior to the catastrophe in the model. This provides encouragement for the prospects of earthquake prediction using LURR, and motivates continued study of the LURR mechanism, earthquake forecasting research, and the use of advanced simulation models to probe the physics of earthquakes.

\section{Acknowledgments}

This research was funded by the National Natural Sciences Foundation of China (Grant No. 19732060 and 40004002), the Australian Research Council (ARC), The University of Queensland, and the ARC International Researcher Exchange Scheme 2000 (IREX). Computations were made on the Australian Solid Earth Simulator (ASES) thematic parallel supercomputer facility (phase I - 16 processor SGI Origin 
3800) funded by the ARC, The University of Queensland, CSIRO, University of Western Australia and Silicon Graphics.

\section{REFERENCES}

Bowman, D. D., Oulllon, G., Sammis, C. G., Sornette, A., and Sornette, D. (1998), An Observational Test of the Critical Earthquake Hypothesis, J. Geophys. Res. 103, 24,359-24,372.

Bufe, C. G. and Varnes, D. J. (1993), Predictive Modeling of the Seismic Cycle in the Greater San Francisco Bay Region, J. Geophys. Res. 98, 9,871-9,833.

Mora, P. and Place, D. (1994), Simulation of the Frictional Stick-slip Instability, Pure Appl. Geophys. 143, 61-87.

Mora, P. and Place, D. (1998), Numerical Simulation of Earthquake Faults with Gouge: Towards a Comprehensive Explanation of the Heat Flow Paradox, J. Geophys. Res. 103, 21,067-21,089.

Mora, P. and Place, D. (1999), The Weakness of Earthquake Faults, Geophys. Res. Lett. 26, $123-126$.

Mora, P. and Place, D. (2002), Stress Correlation Function Evolution in Lattice Solid Elasto-dynamic Models of Shear and Fracture Zones and Earthquake Prediction, Pure Appl. Geophys. 159, 2413-2426.

Place, D. and Mora, P. (1999), A Lattice Solid Model to Simulate the Physics of Rocks and Earthquakes: Incorporation of Friction, J. Comp. Phys. 150, 1-41.

Place, D. and Mora, P. (2000), Numerical Simulation of Localisation Phenomena in a Fault Zone, Pure Appl. Geophys. 157, 1821-1845.

Place, D. and Mora, P. (2001), A random lattice solid model for simulation of fault zone dynamics and fracture processes. In Bifurcation and Localisation Theory for Soils and Rocks'99 (eds., Mühlhaus H-B., Dyskin, A. V. and Pasternak, E.), (AA Balkema, Rotterdam/Brookfield. 2001).

Place, D., Lombard, F., Mora, P., and Abe, S. (2002), Simulation of the Micro-physics of Rocks Using LSMearth, Pure Appl. Geophys. 159, 1911-1932.

WeI, Y. J., XIA, M. F., Ke, F. J., YIN, X. C., and BAI, Y. L. (2000), Evolution Induced Catastrophe and its Predictability, Pure Appl. Geophys. 157, 1945-1957.

Yin, X. C., Chen, X. Z., Song, Z. P., and Yin, C. (1995), A New Approach to Earthquake Prediction: The Load/Unload Response Ratio (LURR) theory, Pure Appl. Geophys. 145, 701-715.

Yin, X. C., Wang, Y. C., Peng, K. Y., and BAI, Y. L. (2000), Development of a New Approach to Earthquake Prediction: Load/Unload Response Ratio (LURR) Theory, Pure Appl. Geophys. 157, 2365-2383.

Yin, X. C., Mora, P., Peng, K. Y., Wang, Y. C., and Weatherley, D. (2002), Load-Unload Response Ratio and Accelerating Moment (energy) Release Critical Region Scaling and Earthquake Prediction, Pure Appl. Geophys. 159, 2511-2523.

(Received February 20, 2001, revised June 11, 2001, accepted June 15, 2001)

䗆

To access this journal online:

http://www.birkhauser.ch 